\title{
CDISC SEND Laboratory Animal Species Terminology
}

National Cancer Institute

\section{Source}

National Cancer Institute. CDISC SEND Laboratory Animal Species Terminology. NCI Thesaurus. Code C77808.

A terminology set that includes concepts relevant to CDISC SEND laboratory animal species. 